\title{
Expresión de TGF- $\beta$ como indicio temprano de lesión del tejido hepático en la enfermedad de hígado graso no alcohólico
}

\author{
Expression of TGF- $\beta$ as an early sign of liver \\ tissue injury in nonalcoholic fatty liver disease
}

\author{
Lina Lambis, José Belisario Solana, Amileth SuÁrez • \\ Cartagena (Colombia)
}

\section{Resumen}

Introducción: la enfermedad de hígado graso no alcohólico (NAFLD) constituye un problema de salud pública asociado con el síndrome metabólico; su patogénesis implica el inicio de una cascada de señalización bioquímica compleja y su estimulación continua podría consolidar un proceso de fibrogénesis en el tejido. El objetivo del estudio fue analizar expresión de genes implicados en daño hepático, en los procesos iniciales de la lesión en pacientes con NAFLD o con factores de riesgo relacionados a esta patología, en búsqueda de biomarcadores moleculares útiles a la práctica clínica tales como TGF- $\beta 1$, COL1A2 y MMP20.

Metodología: estudio analítico de corte transversal. Se estudiaron características epidemiológicas, bioquímicas, y expresión génica de TGF- $\beta 1$, COL1A2 y MMP20 en tejido hepático, en individuos con factores de riesgo para NAFLD.

Resultados: se incluyeron 83 participantes con factores de riesgo asociados a NAFLD, 22 individuos $(26.5 \%)$ fueron diagnosticados con NAFLD mediante ultrasonografía. Los factores de riesgo hallados fueron hipertensión arterial $(50.6 \%)$, obesidad $(49.4 \%)$, diabetes mellitus (34.9\%) y dislipidemia $(21.7 \%)$. La dislipidemia fue significativamente asociada con el riesgo de desarrollar $\operatorname{NAFLD}(O R=4 ; p=0.011)$. Se encontraron diferencias significativas para colesterol total $(p<0.05)$; y una expresión génica de TGF- $\beta 1$ (con NAFLD $p<0.0001$ y sin NAFLD $p<0.0001$ frente al control) y COL1A2 (con NAFLD $p=0.002$ y sin NAFLD $p=0.955$ frente al control) con un patrón de expresión creciente a mayor grado de lesión hepática.

Conclusión: para concluir, sugerimos activación de las vías de señalización que conducen a fibrogénesis en individuos con factores de riesgo para NAFLD, y mucho más acentuada en pacientes con NAFLD. (Acta Med Colomb 2018; 43: 142-149).

Palabras clave: hígado graso de origen no alcohólico (NAFLD), factor de crecimiento transformante $\beta$ 1, colágeno 1 alfa 2, metaloproteasa de matriz 20, fibrogénesis.

\footnotetext{
Abstract

Introduction: nonalcoholic fatty liver disease (NAFLD) is a public health problem associated with the metabolic syndrome; its pathogenesis implies the start of a complex biochemical signaling cascade and its continuous stimulation could consolidate a fibrogenesis process in the tissue. The aim of the study was to analyze expression of genes involved in liver damage in the initial processes of the lesion in patients with NAFLD or with risk factors related to this pathology, in search of molecular biomarkers useful to clinical practice such as TGF- $\beta 1$, COL1A2 and MMP20.

Methodology: cross-sectional analytical study. Epidemiological, biochemical, and gene expression characteristics of TGF- $\beta 1$, COL1A2 and MMP20 in liver tissue in individuals with risk factors for NAFLD were studied.

Results: 83 participants with risk factors associated to NAFLD were included; 22 individuals $(26.5 \%)$ were diagnosed with NAFLD by ultrasonography. The risk factors found were hypertension (50.6\%), obesity (49.4\%), diabetes mellitus (34.9\%) and dyslipidemia (21.7\%). Dyslipidemia was significantly associated with the risk of developing NAFLD $(O R=4 ; p=0.011)$. Significant
}

Dra. Lina Lambis Anaya: Investigadora Bacterióloga. Magister en Bioquímica. Grupo Prometeus \& Biomedicina aplicada a las ciencias clínicas, Laboratorio de Bioquímica, Facultad de Medicina, Universidad de Cartagena; Dr. José Belisario Solana Tinoco: Especialista en Cirugía General y del Aparato Digestivo, Unidad de Hígado Vías Biliares, Hospital Universitario del Caribe - Cartagena, Liga Contra el Cáncer; Amileth Suárez Causado: Doctora en Bioquímica y Biología Molecular. Grupo Prometeus \& Biomedicina Aplicada a las Ciencias Clínicas, Docente de Bioquímica $(\mathrm{PhD})$, Facultad de Medicina, Universidad de Cartagena. Cartagena (Colombia). Correspondencia: Dra. Amileth Suárez Causado, Cartagena (Colombia).

E-mail: asuarezc1@unicartagena.edu.co Recibido: 23/III/2017 Aceptado: 25/VI/2018 
differences were found for total cholesterol ( $\mathrm{p}<0.05$ ); and a gene expression of TGF- $\beta 1$ (with NAFLD $\mathrm{p}<0.0001$ and without NAFLD $\mathrm{p}<0.0001$ versus control) and COL1A2 (with NAFLD $\mathrm{p}$ $=0.002$ and without NAFLD $\mathrm{p}=0.955$ versus control) with a pattern of increasing expression at higher degree of liver injury.

Conclusion: to conclude, we suggest activation of the signaling pathways that lead to fibrogenesis in individuals with risk factors for NAFLD, and much more accentuated in patients with NAFLD. (Acta Med Colomb 2018; 43: 142-149).

Keywords: fatty liver of non-alcoholic origin (NAFLD), transforming growth factor $\beta$ 1, collagen 1 alpha 2, matrix 20 metalloprotease, fibrogenesis.

\section{Introducción}

La enfermedad de hígado graso no alcohólico (NAFLD del Inglés Non-Alcoholic Fatty Liver Disease), se define como la acumulación excesiva de grasa en los hepatocitos en un porcentaje mayor de $10 \%$ del peso del hígado en individuos con ausencia de consumo significativo de alcohol, incluye un amplio espectro patológico que puede ir desde la simple esteatosis a esteatohepatitis, fibrosis, cirrosis y cáncer hepático (1-3). Existe una correlación entre NAFLD y factores de riesgo como obesidad, diabetes, dislipidemia, hipertensión arterial (HTA), y otras condiciones relacionadas al estilo de vida (4).

El tratamiento de NAFLD se ha direccionado a pérdida de peso más terapia farmacológica para mejorar resistencia a insulina o dislipidemia, también cirugías bariatricas han resultado efectivas; en tratamiento farmacológico, actualmente emergen datos de un ensayo clínico con Oltipraz (sintético de ditioltiona) como inhibidor de genes lipogénicos que mejora el hígado graso e inhibe su progresión a fibrosis hepática (5).

La lesión molecular en NAFLD se presenta por el acumulo de grasa en el tejido e inicia una cascada de señalización bioquímica que incluye estrés oxidativo, respuesta inflamatoria, disfunción mitocondrial, peroxidación de lípidos y disfunción del equilibrio de la matriz extracelular; estas señales con la estimulación continua a través del tiempo consolidan en un proceso de fibrogénesis en el tejido, eventos en los que el factor de crecimiento transformante $\beta 1$ (TGF- $\beta 1$ ) juega un papel fundamental. Este último, es identificado como una importante citoquina profibrotica que promueve la expresión de colágeno I por parte de las células estrelladas y se halla posiblemente implicado en la inhibición de metaloproteinasas de matriz (MMP) (6-8). Los estudios moleculares en etapas iniciales de lesión del tejido son de gran ayuda a la identificación de nuevos biomarcadores moleculares que aporten mayor información acerca del grado de lesión del hepatocito, contribuyendo con los actuales métodos de diagnóstico y pronóstico. Se han realizado algunos estudios moleculares en otros países con poblaciones diferentes a nuestras condiciones y adicionalmente mucha de la información existente es dada por modelos animales, por tanto la relevancia del trabajo $(9,10)$.

El objetivo del estudio fue analizar expresión de genes implicados en daño hepático en los procesos iniciales de la lesión en pacientes con NAFLD o con factores de riesgo relacionados a la esteatosis hepática, en búsqueda de biomarcadores moleculares útiles a la práctica clínica. Encontramos que los individuos con factores de riesgo a NAFLD y aún más en la esteatosis hepática se expresa la citoquina TGF $\beta$, lo cual activa cascadas de señalización claves para progresión a fibrosis y HCC (Hepatocarcinoma).

\section{Material y métodos}

Estudio analítico de corte transversal, en el que se estudiaron características epidemiológicas, bioquímicas y se evaluó la expresión génica de TGF- $\beta 1$, COL1A2 y MMP20 en tejido hepático, y su posible asociación con la presencia de NAFLD.

El estudio se realizó con los pacientes con factores de riesgo para NAFLD que acudieron al servicio de Hígado y Vías Biliares del Hospital Universitario del Caribe (Cartagena-Colombia), durante 15 meses consecutivos.

Se excluyeron pacientes cuyo consumo de alcohol excediera los $20 \mathrm{~g}$ /día para hombres y $10 \mathrm{~g}$ /día para mujeres, antecedentes personales de hepatitis viral, medicamentos hepatotóxicos, hemocromatosis y enfermedad de Wilson. Se recopilaron datos sociodemográficos (edad, genero, procedencia), antropométricos y clínicos (peso, talla, índice de masa corporal, factores de riesgo para NAFLD) de cada participante después de su consentimiento informado.

\section{Determinaciones bioquímicas}

Para la realización de las mediciones bioquímicas se recolectaron muestras de sangre periférica por punción de una vena de la zona anterior del brazo o dorso de la mano en tubos con heparina sódica como anticoagulante (BD Vacutainer $\left.{ }^{\circledR}\right)$ mediante sistema de tubos al vacío. Se determinaron concentraciones plasmáticas de los siguientes analitos teniendo en cuenta los valores de referencia: glucosa $(70-110 \mathrm{mg} / \mathrm{dL})$, colesterol total (hasta $200 \mathrm{mg} / \mathrm{dL}$ ), triglicéridos (hasta 150 $\mathrm{mg} / \mathrm{dL}$ ), bilirrubinas (total hasta $1,1 \mathrm{mg} / \mathrm{dL}$; directa hasta $0.25 \mathrm{mg} / \mathrm{dL}$; indirecta hasta $0.76 \mathrm{mg} / \mathrm{dL}$ ), albumina (3.4-4.8 $\mathrm{g} / \mathrm{dL}$ ) y creatinina (hombres $0.6-1.1 \mathrm{mg} / \mathrm{dL}$; mujeres $0.5-1.0$ $\mathrm{mg} / \mathrm{dL}$ ), por los métodos glucosa oxidasa, colesterol oxidasa, glicerol fosfato oxidasa, sulfanílico diazoado, verde de bromocresol y picrato alcalino respectivamente; se determinó la actividad enzimática de aspartato aminotransferasa - AST 
(hasta $40 \mathrm{U} / \mathrm{L}$ ), alanino aminotransferasa - ALT (hasta 41 U/L), gamma glutamil transferasa - GGT (hombres $<50$ $\mathrm{U} / \mathrm{L}$; mujeres $<30 \mathrm{U} / \mathrm{L}$ ) y fosfatasa alcalina (hombres $<270$ $\mathrm{U} / \mathrm{L}$; mujeres $<240 \mathrm{U} / \mathrm{L}$ ) por los métodos de la IFCC (International Federation of Clinical Chemistry), empleando los Kit de Biosystems ${ }^{\circledR}$ correspondiente en cada caso. Todas las mediciones se realizaron siguiendo las recomendaciones del fabricante. Los valores correspondientes a recuento de plaquetas $\left(150000-450000 / \mathrm{mm}^{3}\right)$ y pruebas de coagulación: tiempo de protrombina (9.7 - 14 segundos), tiempo parcial de tromboplastina (23.5-34.3 segundos) e INR, fueron consultados de sus historias clínicas.

\section{Detección de hígado graso en los individuos de estudio}

A los participantes del estudio (pacientes con factores de riesgo para NAFLD) se les realizó una ultrasonografía abdominal total con ecógrafo SonoAce 8000 Live (Medison CO.,Ltd.) para determinar diagnóstico de NAFLD, adicional del examen clínico.

De los 83 participantes, 22 fueron programados previamente para biopsia hepática pero sólo 10 aceptaron el consentimiento informado para destinar una parte de la misma para el estudio molecular, las cuales fueron dadas por patología y congeladas para posterior extracción de ARN, este aspecto limitó el número de muestras recolectadas para el análisis molecular. Todos los procedimientos realizados en el estudio se llevaron a cabo de acuerdo con los principios expresados en la declaración de Helsinki (11) y fueron aprobados por el comité de ética de la Universidad de Cartagena.

\section{Preparación ARN: extracción de ARN, síntesis de cDNA y RT-PCR}

El ARN total fue extraído de 0.02 gramos de tejido hepático usando RNeasy MiniKit ${ }^{\circledR}$ (QIAGEN, Valencia, CA) y tratados por DNA-free ${ }^{\mathrm{TM}}$ (Ambion, Austin, Tx) siguiendo las consideraciones del fabricante. Abundancia y ratio entre $28 \mathrm{~S}$ y $18 \mathrm{~S}$ rARN fue monitoreado por electroforesis en gel de agarosa. Para la cuantificación del ácido nucleico se utilizó el espectrofotómetro Nanodrop ${ }^{\mathrm{TM}}$ (Thermo Fisher Scientific, USA), midiendo a absorbancia 230, 260 y $280 \mathrm{~nm}$.

Para obtención de cDNA se utilizó el kit QuantiTect ${ }^{\circledR}$ Reverse Transcription (QIAGEN, Valencia, CA) siguiendo las indicaciones del fabricante. La amplificación se realizó por RT-PCR utilizando los cebadores TGF- $\beta 1$ (for GGCAGCTGTACATTGACTTCC, rev CCTTGCTGTACTGCGTGTCC), COL1A2 (for CTGGTAGTCGTGGTGCAAGT, rev AATGTTGCCAGGCTCTCCTC), MMP-20 (for TCGAGGTGGACAAAGCAGTG, rev TGGGCTTCCCCAGGAATACT), y GUSB (for ATCACCGTCACCACCAGCGT, rev GTCCCATTCGCCACGACTTTGT) (Operon-Eurofins, Huntsville, AL, USA) como gen control. Las condiciones de la PCR fueron las siguientes: 5 minutos a $95^{\circ} \mathrm{C}$, seguido de 35 ciclos de $94^{\circ} \mathrm{C}$ por $0.5-1$ minuto, $60-62^{\circ} \mathrm{C}$ durante 30 segundos, $72^{\circ} \mathrm{C}$ durante $0.5-1$ minuto y elongación final a $72^{\circ} \mathrm{C}$ por 10 minutos. Los productos amplificados fueron separados mediante electroforesis con gel de agarosa al $1.0 \%$ teñidos con EZ Vision ${ }^{\circledR}$ (Amresco, OH, USA) para visualización en el equipo ChemiDoc ${ }^{\mathrm{TM}} \mathrm{XRS}+$ System (BIORAD, CA, USA). La expresión relativa de los genes se analizó por densitometría usando el software de análisis de imagen unidimensional QuantityOne ${ }^{\circledR}$ (BioRad, CA, USA). Cada experimento se realizó por triplicado independientemente.

\section{Análisis estadístico}

Se inició mediante el diseño de una base de datos en Excel Microsoft ${ }^{\circledR}$ office 2010 en la que se tabularon los datos de los participantes, posteriormente ésta fue transportada al programa STATA ${ }^{\circledR}$ (Stata Corp. LP, College Station, TX, USA). Para el análisis descriptivo fueron usadas las medidas de tendencia central, dispersión y proporciones. Inicialmente se evaluó el supuesto de normalidad de los datos a partir del test de Kolmogorov-Smirnov. Para analizar la asociación entre las variables se utilizó la prueba Chi-cuadrado para las variables cualitativas y la prueba t-student para variables cuantitativas, para distribuciones no paramétricas se utilizó la prueba de Wilcoxon-Mann-Whitney; además se calculó Odds ratio. Para todas las pruebas se asumió una probabilidad límite de decisión de 0.05 .

\section{Resultados}

\section{Características de la población de estudio}

De 98 individuos identificados con factores de riesgo para NAFLD, 83 aceptaron su participación en el estudio. A todos los participantes se les realizó ultrasonografía abdominal total para el diagnóstico de NAFLD, a partir de características cualitativas que incluyeron ecogenicidad, ecotextura y visibilidad de los vasos, dividiendo la población de estudio en los grupos: con NAFLD para aquellos en donde se confirmó diagnóstico de NAFLD por ultrasonografía, y sin NAFLD en aquellos que resultaron negativo a hígado graso en el momento del estudio. De los 83 participantes, se confirmó el padecimiento de NAFLD en 22 individuos (26.5\%), mientras en los 61 individuos restantes con factores de riesgo para NAFLD (73.5\%) no se evidenció hígado graso en el momento del estudio.

En cuanto a las variables sociodemográficas en el total de los individuos estudiados, se encontró una distribución mayoritariamente representada por el género femenino (75.9\%), el promedio de edad fue de $55.4(\mathrm{DE}=18.53)$ y una procedencia mayoritaria del área urbana (72.3\%). Una vez clasificados los participantes por grupos (con NAFLD y sin NAFLD), persistió un mayor porcentaje del género femenino en ambos grupos (63.64\% en el grupo con NAFLD y $80.33 \%$ en el grupo sin NAFLD), e igualmente una procedencia con mayor representación en el área urbana tanto en el grupo con NAFLD (72,73\%) como en el grupo sin NAFLD (72.13\%). En el total de los participantes se halló un peso y talla promedio igual a $77.2 \mathrm{Kg}$ y 1.62 metros respectivamente, para una media del IMC de $29.86 \mathrm{Kg} / \mathrm{m}^{2}$, medidas antropométricas que al analizarse por grupos mostraron un índice de masa 
Tabla 1. Distribución de las características sociodemográficas y antropométricas de los participantes.

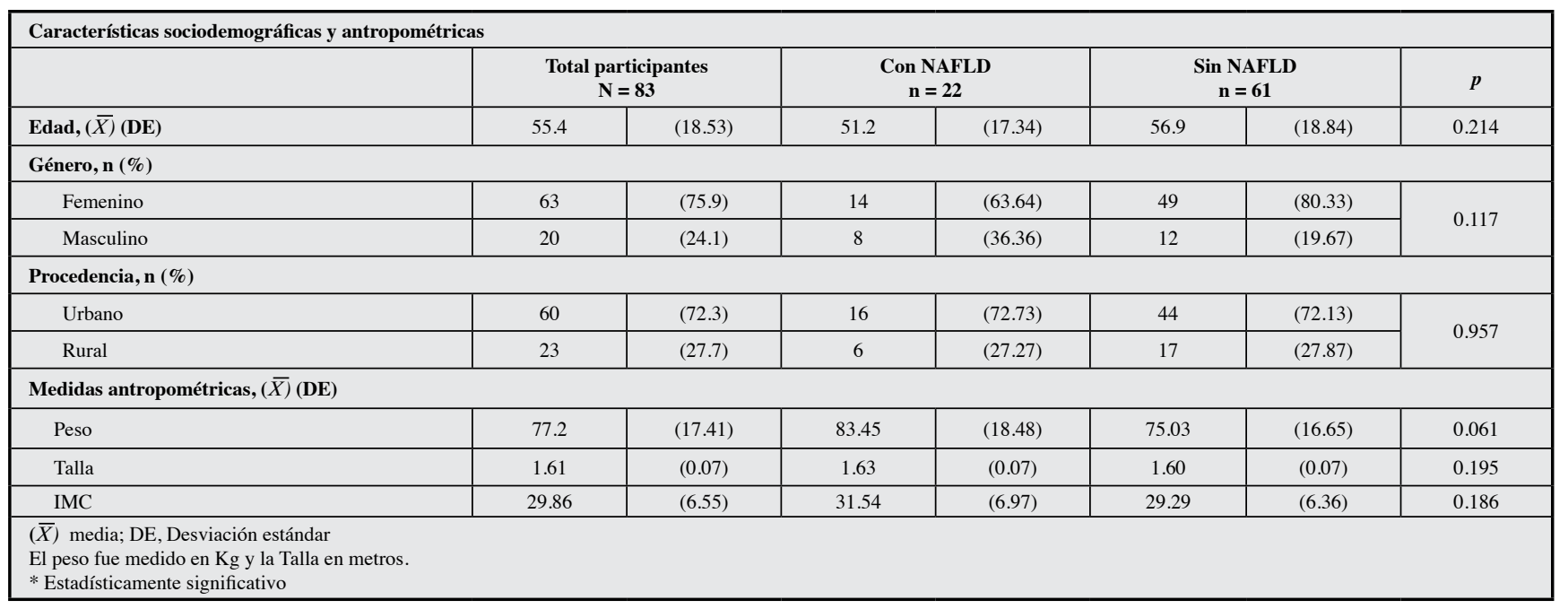

corporal (IMC) promedio igual a $31.54 \mathrm{Kg} / \mathrm{m}^{2}(\mathrm{DE}=6.97)$ en individuos con NAFLD, y $29.29 \mathrm{Kg} / \mathrm{m}^{2}$ (DE=6.36) en individuos sin NAFLD. La Tabla 1 muestra comparaciones de las características sociodemográficas y antropométricas entre los grupos diagnosticados con NAFLD y el grupo con factores de riesgo de la enfermedad sin NAFLD.

Los factores de riesgo hallados en la población de estudio están relacionados con el síndrome metabólico, los cuales fueron hipertensión arterial (HTA) (50.6\%), obesidad (49.4\%), y finalmente el padecimiento de diabetes mellitus (34.9\%) y dislipidemia (21.7\%). La Tabla 2 muestra las diferencias de los cuatro factores de riesgo asociados a NA-
FLD (obesidad, diabetes mellitus, dislipidemia, hipertensión arterial) entre los grupos con/sin la condición de NAFLD. La dislipidemia fue significativamente asociada con el riesgo de desarrollar NAFLD $(O R=4 ; p=0.011)$. Los marcadores bioquímicos plasmáticos evaluados, mostraron de forma general en la totalidad de participantes, un comportamiento de la mediana dentro de los valores de referencia establecidos para estas mediciones, sólo se observaron valores por encima del rango considerado normal para gamma glutamil transferasa $(\mathrm{Me}=201 \mathrm{U} / \mathrm{L})$ y fosfatasa alcalina $(\mathrm{Me}=307 \mathrm{U} / \mathrm{L})$.

Por su parte, al observar los datos hallados por grupos, se encontraron valores de transaminasas AST $(\mathrm{Me}=49.5)$ y

Tabla 2. Factores de riesgo asociados con el desarrollo de NAFLD.

\begin{tabular}{|c|c|c|c|c|c|}
\hline \multicolumn{6}{|c|}{ Factores de riesgo asociados } \\
\hline & $\begin{array}{c}\text { Total participantes } \\
\mathrm{N}(\%)\end{array}$ & $\begin{array}{c}\text { Con NAFLD } \\
\text { n (\%) }\end{array}$ & $\begin{array}{l}\text { Sin NAFLD } \\
\text { n }(\%)\end{array}$ & \multirow{2}{*}{$\begin{array}{c}\text { OR } \\
\text { (IC 95\%) }\end{array}$} & \multirow{2}{*}{$p$} \\
\hline & $83(100)$ & $22(26.5)$ & $61(73.5)$ & & \\
\hline \multicolumn{6}{|c|}{ Obesidad } \\
\hline Sí & $41(49.4)$ & $12(54.5)$ & $29(47.5)$ & \multirow{2}{*}{$\begin{array}{c}1.32 \\
(0.45-3.98)\end{array}$} & \multirow{2}{*}{0.573} \\
\hline No & $42(50.6)$ & $10(45.5)$ & $32(52.5)$ & & \\
\hline \multicolumn{6}{|c|}{ Diabetes mellitus } \\
\hline Sí & $29(34.9)$ & $8(36.4)$ & $21(34.4)$ & \multirow{2}{*}{$\begin{array}{c}1.09 \\
(0.34-3.33)\end{array}$} & \multirow{2}{*}{0.870} \\
\hline No & $54(65.1)$ & $14(63.6)$ & $40(65.6)$ & & \\
\hline \multicolumn{6}{|c|}{ Dislipidemia } \\
\hline Sí & $18(21.7)$ & $9(40.9)$ & $9(14.8)$ & \multirow{2}{*}{$\begin{array}{c}4 \\
(1.13-13.85)\end{array}$} & \multirow{2}{*}{$0.011^{*}$} \\
\hline No & $65(78.3)$ & $13(59.1)$ & $52(85.2)$ & & \\
\hline \multicolumn{6}{|c|}{ Hipertensión arterial } \\
\hline Sí & $42(50.6)$ & $7(31.8)$ & $35(57.4)$ & \multirow{2}{*}{$\begin{array}{c}0.35 \\
(0.11-1.07)\end{array}$} & \multirow{2}{*}{$0.039 *$} \\
\hline No & $41(49.4)$ & $15(68.2)$ & $26(42.6)$ & & \\
\hline
\end{tabular}


ALT $(\mathrm{Me}=76)$ por encima de los valores normales en los individuos Con NAFLD, a diferencia de los individuos Sin NAFLD, cuyos valores para estas enzimas (AST Me=29; ALT $\mathrm{Me}=27$ ) fueron menores y se comportaron dentro del rango de referencia. Igualmente, los niveles de fosfatasa alcalina en el grupo con NAFLD se hallaron por fuera del rango de referencia y fueron superiores al compararlos con los valores hallados en el grupo sin NAFLD, sin embargo en este estudio no se encontraron diferencias significativas entre ambos grupos para estas mediciones.

En cuanto a las mediciones de colesterol total y triglicéridos, se observaron valores mayores para estos dos analitos en individuos con NAFLD, colesterol total con una mediana de $193.2 \mathrm{mg} / \mathrm{dL}$, valor que se ubica dentro del rango normal, y triglicéridos con mediana por encima de los valores normales e igual a $173.7 \mathrm{mg} / \mathrm{dL}$, al comparar por grupos sólo se hallaron diferencias significativas para colesterol total $(p<0.05)$ (Tabla 3).

\section{Expresión diferencial de genes}

Fue evaluada la expresión de genes relacionada con el proceso fibrogénico, en los pacientes con NAFLD y el grupo con factores de riesgo (sin NAFLD). Se estudiaron diez muestras, porque fueron los individuos que aceptaron ceder las muestras por parte de la unidad de patología. Los genes analizados fueron el factor de crecimiento transformante beta 1 (TGF- $\beta 1$ ), colágeno 1 alfa 2 (Col1A2), metaloproteinasa 20 (MMP20), y en las condiciones del experimento el nivel de ARNm hepático fue detectado.

El ARNm de TGF- $\beta 1$ (con NAFLD vs control: $p<0.0001$; sin NAFLD vs control: $\mathrm{p}<0.0001)$ y el ARNm de COL1A2 (con NAFLD vs control: $p=0.002$; sin NAFLD vs control: $p=0.955$ ) mostraron un patrón de expresión creciente a mayor grado de lesión hepática, en hepatocitos del control sano no se observó expresión del ARNm (excepción para COL1A2), mientras que en los tejidos hepáticos de los individuos con factores de riesgo, como los pacientes diagnosticados con NAFLD se observó expresión de los genes, siendo mayor la de este último. El ARNm de MMP20 se mantuvo sin modulación (sin expresión del gen) en todas las condiciones del experimento (con NAFLD vs control: $p=0.068$; sin NAFLD vs Control: $p=0.181$ ). Se utilizó GUSB como gen normalizador para cada gen analizado, se realizaron tres experimentos de PCR independientemente (Figura 1).

\section{Discusión}

La enfermedad de hígado graso tiene una creciente prevalencia a nivel global en los últimos años, con riesgo de incremento por factores de riesgo como la obesidad, diabetes, hipertensión arterial, con lo cual es extensamente estudiada a nivel mundial por ser un problema de salud pública. Los estudios moleculares en NAFLD se han realizado en su mayoría en modelos animales, y han involucrado TGF- $\beta$ como una citoquina con un papel crucial en la fibrogénesis en la enfermedad hepática crónica, se ha observado una señalización persistente que resulta en una desregulación de mecanismos normales de síntesis - degradación de matriz extracelular y por ende en su excesiva acumulación $(12,13)$.

Este estudio analizó expresión de genes relacionados con el proceso fibrogénico en los procesos iniciales de la lesión en individuos con NAFLD y en individuos con factores de

Tabla 3. Niveles plasmáticos de los parámetros bioquímicos estudiados en los participantes.

\begin{tabular}{|c|c|c|c|c|c|c|c|}
\hline \multicolumn{8}{|l|}{ Mediciones bioquímicas } \\
\hline \multirow[b]{2}{*}{$\operatorname{ALT}(\mathrm{U} / \mathrm{L})$} & \multicolumn{2}{|c|}{$\begin{array}{c}\text { Total participantes } \\
\text { Me [RI] }\end{array}$} & \multicolumn{2}{|c|}{$\begin{array}{c}\text { Con NAFLD } \\
\text { Me [RI] }\end{array}$} & \multicolumn{2}{|c|}{$\begin{array}{c}\text { Sin NAFLD } \\
\text { Me [RI] }\end{array}$} & \multirow{2}{*}{$\begin{array}{c}p \\
0.073\end{array}$} \\
\hline & 38 & [20 - 129] & 76 & [28-133] & 27 & [19-105] & \\
\hline $\operatorname{AST}(\mathrm{U} / \mathrm{L})$ & 38 & [19- 70] & 49.5 & [19-92.5] & 29 & [20-67] & 0.356 \\
\hline AST/ALT & 1.0 & {$[0.75-1.29]$} & 0.84 & {$[0.53-1.24]$} & 1.05 & {$[0.83-1.30]$} & 0.116 \\
\hline Bilirrubina total (mg/dL) & 0.90 & {$[0.47-2.80]$} & 0.74 & {$[0.47-1.41]$} & 0.91 & {$[0.535-2.95]$} & 0.967 \\
\hline Bilirrubina directa $(\mathrm{mg} / \mathrm{dL})$ & 0.46 & {$[0.19-2.11]$} & 0.51 & {$[0.28-1.15]$} & 0.41 & [0.185-1.97] & 0.561 \\
\hline Bilirrubina indirecta $(\mathrm{mg} / \mathrm{dL})$ & 0.34 & {$[0.16-0.60]$} & 0.23 & {$[0.18-0.4]$} & 0.38 & {$[0.18-0.65]$} & 0.256 \\
\hline GGT (U/L) & 201 & {$[52-384]$} & 136 & [54-146] & 258 & {$[47-586]$} & 0.671 \\
\hline Albumina $(\mathrm{g} / \mathrm{dL})$ & 3.29 & {$[2.70-3.50]$} & 3.42 & [3.16-3.925] & 3.17 & {$[2.61-3.43]$} & 0.172 \\
\hline TP (segundos) & 10.7 & {$[9.9-12.2]$} & 10.5 & [10.3-14.1] & 10.7 & [10-11.9] & 0.416 \\
\hline TPT (segundos) & 25.7 & {$[22.8-29.5]$} & 25.35 & [21.9-27.05] & 26 & [23.1-29.9] & 0.336 \\
\hline INR & 1.00 & [0.93-1.10] & 1.04 & [0.96-1.24] & 1 & [0.935-1.07] & 0.311 \\
\hline Glicemia & 99.5 & [85.1-121.9] & 108.4 & {$[85.7-131.4]$} & 98.3 & {$[85.1-120.7]$} & 0.348 \\
\hline Colesterol total $(\mathrm{mg} / \mathrm{dL})$ & 168 & [139-202] & 193.2 & {$[170.3-214.2]$} & 159.7 & {$[137,4-194.5]$} & $0.009 *$ \\
\hline Triglicéridos (mg/dL) & 152 & [133 - 199] & 173.7 & {$[133,9-243.3]$} & 151.77 & [133.8 -189.9] & 0.217 \\
\hline Fosfatasa alcalina (U/L) & 307 & [217 - 469] & 376 & {$[275-477.5]$} & 279 & {$[208-468]$} & 0.452 \\
\hline Creatinina & 0.84 & {$[0.69-1.01]$} & 0.755 & {$[0.55-1.05]$} & 0,86 & {$[0.75-0.97]$} & 0.166 \\
\hline Plaquetas & 322500 & [239250 - 428750] & 317000 & {$[258500-430000]$} & 325000 & {$[239500-425000]$} & 0.918 \\
\hline
\end{tabular}


A.

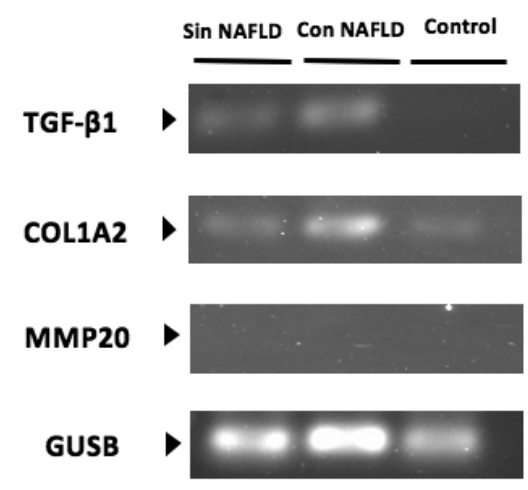

C

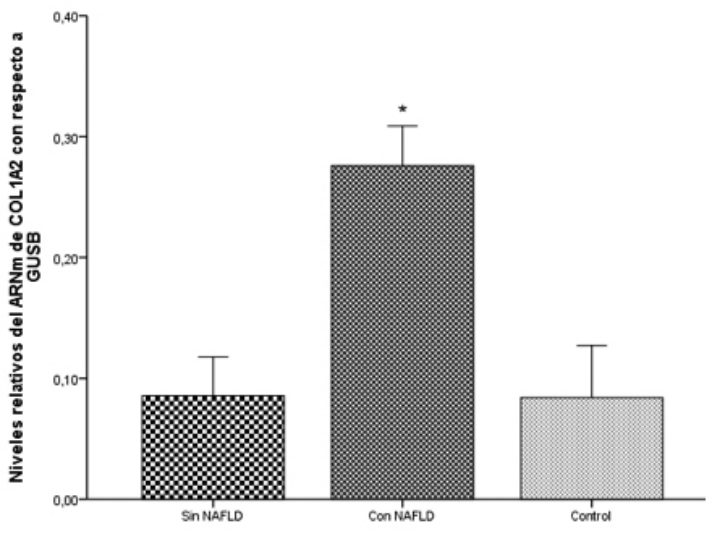

B.

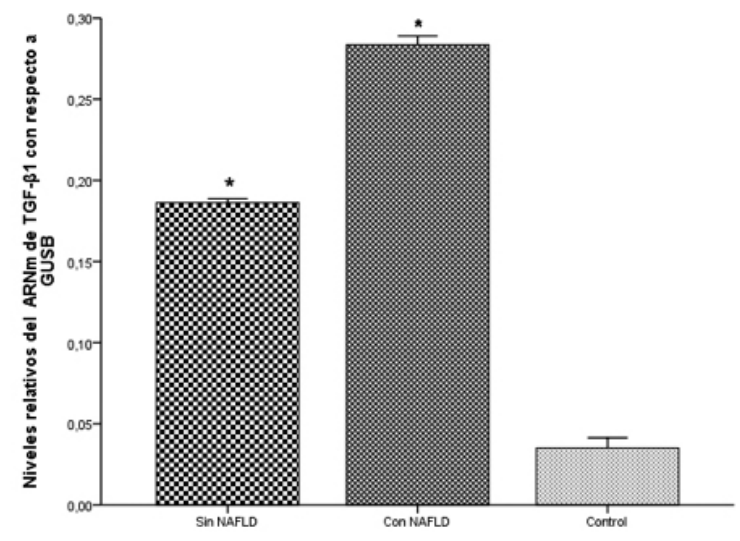

D.

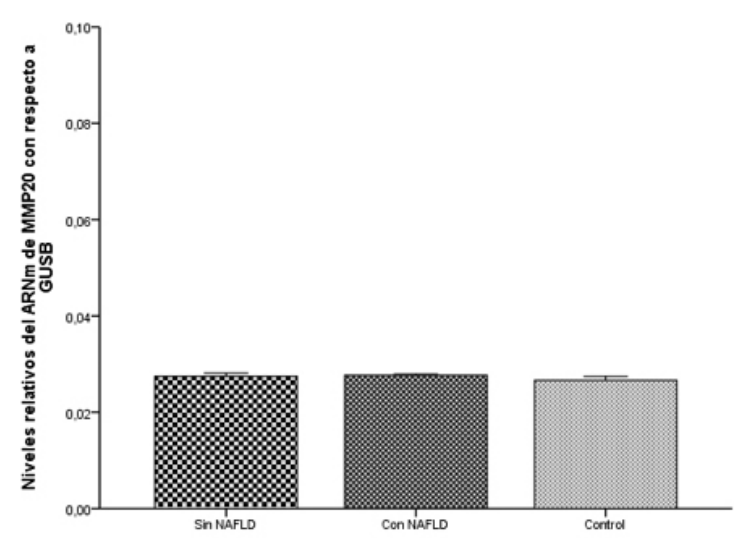

Figura 1. Expresión génica de TGF- $\beta 1, C O L 1 A 2$ Y MMP20 mediante amplificación por PCR convencional en tejido hepático de individuos con factores de riesgo sin desarrollo de NAFLD, de individuos con NAFLD y en control sano. Se utiliza GUSB como gen de referencia. A. Amplificación por PCR en geles de agarosa 1\% de TGF- $\beta 1$, COL1A2 Y MMP20. B. Niveles de expresión relativa del ARNm de TGF- $\beta 1$ con respecto a GUSB por grupos. C. Niveles de expresión relativa del ARNm de COL1A2 con respecto a GUSB por grupos. D. Niveles de expresión relativa del ARNm de MMP20 con respecto a GUSB por grupos. Los asteriscos indican valores de p<0,05 al comparar los grupos Sin NAFLD / Con NAFLD frente al control.

riesgo relacionados a NAFLD en búsqueda de biomarcadores útiles en la práctica clínica.

En las características sociodemográficas, se observó en el total de los individuos mayor proporción del género femenino procedentes del área urbana, comportamiento que se mantuvo al clasificar a los individuos de estudio por grupos según el diagnóstico ecográfico (con NAFLD o sin NAFLD); este comportamiento es muy similar a estudios previos $(14,15)$. Al igual que otros autores, la procedencia del área urbana, ha permitido confirmar que la urbanización ha incrementado la frecuencia de trastornos como la diabetes mellitus, obesidad, dislipidemia e HTA(16-19), posiblemente debido a hábitos de vida poco saludables que conllevan también a IMC relevantes, tales como los valores hallados en este estudio. El índice de masa corporal de los individuos en estudio es una variable que muchos autores también han utilizado para estimar la obesidad aún con sus limitaciones, además algunos informes demuestran la asociación entre un mayor índice de masa cor- poral (IMC), la diabetes y el riesgo de padecer hígado graso no alcohólico $(20,21)$.

Confirmamos, la hipertensión arterial como el factor de riesgo más frecuente en los pacientes con NAFLD tal como se ha documentado (22), seguido de obesidad, diabetes mellitus y dislipidemia significativa, en concordancia con lo reportado en otros estudios $(4,23-28)$. Estos factores de riesgo, de alguna manera conllevan a resistencia a la insulina, cuyos mecanismos bioquímicos incluyen desregulación en la secreción de adipoquinas, disminuyendo así la adiponectina e incrementando la secreción de TNFa (citoquina que promueve la resistencia a la insulina local y sistémica), esto conlleva a inhibición de la lipolisis del triacilglicerol y favorece el flujo de ácidos grasos no esterificados al hígado consecuente a la excesiva acumulación hepática de lípidos $(3,29,30)$. La presencia de hiperinsulinemia o resistencia a la insulina y la asociación con algunas de las características del síndrome metabólico, confirma que NAFLD constituye 
el componente hepático del mismo, pero en el estudio no se consideró la contribución relativa de cada componente del síndrome metabólico al riesgo de NAFLD (31). El colesterol total plasmático fue significativo al comparar entre los grupos estudiados, contrario a los resultados reportados por Sepeliotes y col. en individuos seleccionados a partir del Framingham Heart Study, que no hallaron valores de colesterol que condujeran a relacionar los niveles altos de este metabolito con presentar NAFLD (32). Por su parte, Shen y col. y Kim y col., al comparar individuos con y sin diagnóstico de NAFLD encuentran diferencias significativas tanto para colesterol total como para triglicéridos $(33,34)$. En referencia a las transaminasas, nuestro estudio no mostró diferencias significativas en los grupos estudiados pero, estos valores no garantizan la ausencia de lesión hepática como se ha reportado en la literatura (35-37).

Esta investigación, al igual que otros estudios también presenta limitaciones, la más importante es la aceptación para participar en el estudio molecular por tan sólo 10 individuos. Así mismo, el uso de ultrasonografía para el diagnóstico de esteatosis. La ultrasonografía posee una sensibilidad en la detección de infiltración grasa en el hígado estimada entre 87 y $100 \%$ y una especificidad entre 84 y $89 \%$ (38), pero la biopsia hepática representa mayor sensibilidad pero, esta práctica no es factible ni es éticamente justificado en una condición de bajo riesgo de progresión que no defina tratamiento, por tanto para estos estudios es válido el uso ultrasonografía para definir el grupo de individuos con NAFLD $(39,40)$.

Mostramos que los cambios de expresión de genes TGF- $\beta 1$, COL1A2 fueron más prominentes en el grupo de individuos con NAFLD, sugiriendo desbalance entre acumulo de COL1A2 secretado por las células estrelladas del hígado (HSC) conducente a fibrogénesis, y la regulación a la baja de MMP20 que lo degrada. Un aspecto importante de nuestros resultados es que el grupo de factores de riesgo se comportó de forma similar al grupo con NAFLD, con lo que se podría pensar, que el aumento de la expresión de TGF- $\beta$ en hígado podría ser importante en la progresión de las complicaciones hepáticas y a la progresión a hepatocarcinoma, con lo cual no debe ser desestimada.

Los niveles de expresión de TGF- $\beta 1$ en los individuos con factores de riesgo y aún más en los individuos con NAFLD sugiere una constante señalización por parte de esta citoquina en el tiempo, lo que es de resaltar ya que la estimulación autocrina de TGF- $\beta$ a los miofibroblastos desencadenan activación de la vía Smad, este hecho ha sido observado en enfermedades crónicas hepáticas en modelos animales y podría ser una razón del excesivo efecto del TGF- $\beta$ durante la progresión a fibrosis hepática, con lo cual, el TGF- $\beta$ podría ser un marcador clínico de daño hepático que merece más estudios. Diferentes autores han evaluado la activación de la vía de señalización TGF- $\beta /$ Smad en ratones observando en etapa temprana infiltración de grasa en algunos hepatocitos hasta la presencia evidente de fibrosis, dichos estudios revelaron mayor expresión de Colágeno tipo I $\alpha 1$ y TGF- $\beta 1$ en tejido hepático $(8,41)$. Del mismo modo, Starkel y col. evidenciaron incremento en la expresión de TGF- $\beta 1$ y deposición de colágeno en tejido hepático de ratones con esteatohepatitis (42). La expresión de COL1A2 confirma aún más, la acción del TGF- $\beta$, y sugiere una evidencia de lo que se ha visto en modelos animales de enfermedades crónicas hepáticas, en los cuales se ha demostrado la localización de marcadores como procolágenos 1 A2 (43).

$\mathrm{Al}$ igual que los ensayos antes mencionados en modelos animales, Greco y col. y Sookoian y col., evaluaron la expresión génica en tejido hepático humano de pacientes con NAFLD, encontrando genes relacionados con el metabolismo lipídico y genes implicados en la remodelación de la matriz extracelular $(44,45)$. Estos hallazgos permiten confirmar con mayor certeza los resultados obtenidos en nuestro estudio al sugerir la activación de la vía de señalización del TGF- $\beta$ en tejido hepático durante la esteatosis, e incluso, la expresión de TGF- $\beta$ en hepatocitos desde el momento mismo que se presentan factores de riesgo que hacen mucho más probable la aparición de esta patología, estimulando la deposición de mayor cantidad de colágeno y contribuyendo con una posible progresión a fibrosis de no tomar medidas que reviertan dicho proceso.

Por otra parte, la regulación a la baja de la MMP20 en todos los individuos analizados es muy interesante y amerita mayores estudios, ya que estudios han reportado que en las fases tempranas de lesión hepática, se activan HSC caracterizadas por generación de MMPs y degradación local de la matriz normal del hígado y favorecer la regeneración del tejido (46). Pero los datos muestran una regulación a la baja de MMP20, aún en el hígado de los individuos con factores de riesgo, lo que hipotéticamente sugiere que las vías donde participa como proliferación del hepatocito, apoptosis pueden estar afectadas en estos individuos.

A nivel de Cartagena de Indias es el primer estudio molecular de activación temprana de genes implicados en la génesis de la fibrosis en pacientes con NAFLD y con factores asociados en humanos. Los resultados sugieren una activación de las vías de señalización que conducen a fibrogénesis y su posible progresión a hepatocarcinoma en la condición de factores de riesgo para NAFLD (HTA, obesidad, diabetes mellitus), y mucho más acentuada en los pacientes con NAFLD, con lo cual este estudio amerita seguir evaluando las vías moleculares e intervenir en los individuos con factores de riesgo.

\section{Fuentes de Financiación}

Los autores declaran financiación por plan de Fortalecimiento del grupo Prometeus reconocido por Colciencias por la Universidad de Cartagena, Programa de jóvenes investigadores e innovadores de Colciencias.

\section{Agradecimientos}

Los autores agradecen a la Universidad de Cartagena -Colciencias por apoyar este trabajo a través del programa de Jóvenes investigadores, estudiantes del semillero de investigación del grupo "Prometeus \& Biomedicina aplicada a las ciencias clínicas", a los residentes de la especialidad en cirugía general y radiología de la Universidad de Cartagena, al equipo médico de la unidad de imágenes diagnósticas y de la unidad 
de hígado y vías biliares del hospital Universitario del Caribe, por su valiosa ayuda durante el desarrollo del estudio.

\section{Referencias}

1. Yki-Järvinen H. Non-alcoholic fatty liver disease as a cause and a consequence of metabolic syndrome. Lancet Diabetes Endocrinol. 2014;2(11):901-10.

2. LaBrecque DR, Abbas Z, Anania F, Ferenci P, Khan AG, Goh KL, et al. World Gastroenterology Organisation global guidelines: Nonalcoholic fatty liver disease and nonalcoholic steatohepatitis. J Clin Gastroenterol. 2014;48(6):467-73.

3. Ballestri S, Lonardo A, Bonapace S, Byrne CD, Loria P, Targher G. Risk of cardiovascular, cardiac and arrhythmic complications in patients with nonalcoholic fatty liver disease. World J Gastroenterol. 2014;20(7):1724-45.

4. Koehler EM, Schouten JNL, Hansen BE, van Rooij FJA, Hofman A, Stricker BH, et al. Prevalence and risk factors of non-alcoholic fatty liver disease in the elderly: Results from the Rotterdam study. J Hepatol. 2012;57(6):1305-11.

5. Kim W, Kim BG, Lee JS, Lee CK, Yeon JE, Chang MS, et al. Randomised clinical trial: the efficacy and safety of oltipraz, a liver X receptor alpha-inhibitory dithiolethione in patients with non-alcoholic fatty liver disease. Alimentary pharmacology \& therapeutics. 2017.

6. Louka ML, Ramzy MM. Involvement of fibroblast-specific protein 1 (S100A4) and matrix metalloproteinase-13 (MMP-13) in CCl4-induced reversible liver fibrosis. Gene. 2016;579(1):29-33.

7. Yoshida K, Murata M, Yamaguchi T, Zaki KM. TGF- $\beta /$ Smad signaling during hepatic fibro-carcinogenesis (Review). Int J Oncol. 2014;45(4):1363-71.

8. Yan C, Wang L, Li B, Zhang B-B, Zhang B, Wang Y-H, et al. The expression dynamics of transforming growth factor- $\beta /$ Smad signaling in the liver fibrosis experimentally caused by Clonorchis sinensis. Parasit Vectors. 2015;8:70.

9. Petta S, Valenti L, Bugianesi E, Targher G, Bellentani S, Bonino F, et al. A "systems medicine" approach to the study of non-alcoholic fatty liver disease. Dig Liver Dis. 2016;48(3):333-42.

10. Castera L, Pinzani M. Biopsy and non-invasive methods for the diagnosis of liver fibrosis: does it take two to tango? Gut. 2010;59(7):861-6.

11. World Medical A. World medical association declaration of helsinki: Ethical principles for medical research involving human subjects. JAMA. 2013;310(20):21914.

12. Yang L, Roh YS, Song J, Zhang B, Liu C, Loomba R, et al. TGF- $\beta$ Signaling in Hepatocytes Participates in Steatohepatitis Through Regulation of Cell Death and Lipid Metabolism. Hepatology. 2014;59(2):483-95.

13. Wang Z Z, Yao T, Song Z Z. Extracellular signal-regulated kinases $1 / 2$ suppression aggravates transforming growth factor-betal hepatotoxicity: a potential mechanism for liver injury in methionine-choline deficient-diet-fed mice. Experimental biology and medicine (Maywood, NJ). 2010;235(11):1347-55.

14. Browning JD, Kumar KS, Saboorian MH, Thiele DL. Ethnic differences in the prevalence of cryptogenic cirrhosis. The American journal of gastroenterology. 2004;99(2):292-8.

15. Marchesini G, Bugianesi E, Forlani G, Cerrelli F, Lenzi M, Manini R, et al. Nonalcoholic fatty liver, steatohepatitis, and the metabolic syndrome. Hepatology. 2003;37(4):917-23.

16. Adediran O, Akintunde AA, Edo AE, Opadijo OG, Araoye AM. Impact of urbanization and gender on frequency of metabolic syndrome among native Abuja settlers in Nigeria. J Cardiovasc Dis Res. 2012;3(3):191-6.

17. Pandey RM, Gupta R, Misra A, Misra P, Singh V, Agrawal A, et al. Determinants of urban-rural differences in cardiovascular risk factors in middle-aged women in India: a cross-sectional study. Int J Cardiol. 2013;163(2):157-62.

18. Aschner P, Aguilar-Salinas C, Aguirre L, Franco L, Gagliardino JJ, de Lapertosa SG, et al. Diabetes in South and Central America: An update. Diabetes Res Clin Pract. 2014;103(2):238-43.

19. Aschner P. Epidemiología de la diabetes en Colombia. Av diabetol. 2010;26(2):95100.

20. Loomis AK, Kabadi S, Preiss D, Hyde C, Bonato V, St. Louis M, et al. Body Mass Index and Risk of Nonalcoholic Fatty Liver Disease: Two Electronic Health Record Prospective Studies. The Journal of Clinical Endocrinology and Metabolism. 2016;101(3):945-52.

21. Zelber-Sagi S, Shoham D, Zvibel I, Abu-Abeid S, Shibolet O, Fishman S. Predictors for advanced fibrosis in morbidly obese non-alcoholic fatty liver patients. World Journal of Hepatology. 2017;9(2):91-8.

22. Donati G, Stagni B, Piscaglia F, Venturoli N, Morselli-Labate AM, Rascit $\mathbf{L}$, et al. Increased prevalence of fatty liver in arterial hypertensive patients with normal liver enzymes: role of insulin resistance. Gut. 2004;53(7):1020-3.

23. Portillo-Sanchez P, Bril F, Maximos M, Lomonaco R, Biernacki D, Orsak B, et al. High Prevalence of Nonalcoholic Fatty Liver Disease in Patients With
Type 2 Diabetes Mellitus and Normal Plasma Aminotransferase Levels. J Clin Endocrinol Metab. 2015;100(6):2231-8.

24. Watanabe S, Hashimoto E, Ikejima K, Uto H, Ono M, Sumida Y, et al. Evidence-based clinical practice guidelines for nonalcoholic fatty liver disease/ nonalcoholic steatohepatitis. Hepatol Res. 2015;45(4):363-77.

25. Nascimbeni F, Pais R, Bellentani S, Day CP, Ratziu V, Loria P, et al. From NAFLD in clinical practice to answers from guidelines. J Hepatol. 2013;59(4):85971

26. Jäger S, Jacobs S, Kröger J, Stefan N, Fritsche A, Weikert C, et al. Association between the Fatty Liver Index and Risk of Type 2 Diabetes in the EPIC-Potsdam Study. PLoS One. 2015;10(4):e0124749.

27. Sung K-C, Wild SH, Byrne CD. Resolution of Fatty Liver and Risk of Incident Diabetes. J Clin Endocrinol Metab. 2013;98(9):3637-43.

28. The Korean Association for the Study of the L. KASL clinical practice guidelines: Management of nonalcoholic fatty liver disease. Clin Mol Hepatol. 2013;19(4):325-48

29. Williams KH, Shackel NA, Gorrell MD, McLennan SV, Twigg SM. Diabetes and nonalcoholic Fatty liver disease: a pathogenic duo. Endocr Rev. 2013;34(1):84129

30. Kim D, Choi S-Y, Park EH, Lee W, Kang JH, Kim W, et al. Nonalcoholic Fatty Liver Disease is Associated with Coronary Artery Calcification. Hepatology. 2012;56(2): 10.1002/hep.25593.

31. Marchesini G, Brizi M, Morselli-Labate AM, Bianchi G, Bugianesi E, McCullough AJ, et al. Association of nonalcoholic fatty liver disease with insulin resistance. Am J Med. 1999;107(5):450-5.

32. Speliotes EK, Massaro JM, Hoffmann U, Vasan RS, Meigs JB, Sahani DV, et al. Fatty liver is associated with dyslipidemia and dysglycemia independent of visceral fat: the Framingham Heart Study. Hepatology. 2010;51(6):1979-87.

33. Shen J, Chan HL-Y, Wong GL-H, Choi PC-L, Chan AW-H, Chan H-Y, et al. Non-invasive diagnosis of non-alcoholic steatohepatitis by combined serum biomarkers. J Hepatol. 2012;56(6):1363-70.

34. Kim Y-C, Cho Y-K, Lee W-Y, Kim H-J, Park J-H, Park D-I, et al. Serum adipocyte-specific fatty acid-binding protein is associated with nonalcoholic fatty liver disease in apparently healthy subjects. J Nutr Biochem. 2011;22(3):289-92.

35. Liu X, Hamnvik O-PR, Chamberland JP, Petrou M, Gong H, Christophi CA, et al. Circulating alanine transaminase (ALT) and $\gamma$-glutamyl transferase (GGT), but not fetuin-A, is associated with metabolic risk factors, at baseline and at twoyear follow-up: the prospective Cyprus Metabolism Study. Metabolism: clinical and experimental. 2014;63(6):773-82.

36. Jo SK, Lee WY, Rhee EJ, Won JC, Jung CH, Park CY, et al. Serum gammaglutamyl transferase activity predicts future development of metabolic syndrome defined by 2 different criteria. Clinica chimica acta; international journal of clinical chemistry. 2009;403(1-2):234-40.

37. Mofrad PS, Sanyal AJ. Nonalcoholic fatty liver disease. MedGenMed. 2003;5(2):14.

38. Joseph AEA, Saverymuttu SH, Al-Sam S, Cook MG, Maxwell JD. Comparison of liver histology with ultrasonography in assessing diffuse parenchymal liver disease. Clin Radiol. 1991;43(1):26-31.

39. Saadeh S, Younossi ZM, Remer EM, Gramlich T, Ong JP, Hurley M, et al. The utility of radiological imaging in nonalcoholic fatty liver disease. Gastroenterology. 2002;123(3):745-50.

40. Neuschwander-Tetri BA, Caldwell SH. Nonalcoholic steatohepatitis: summary of an AASLD Single Topic Conference. Hepatology. 2003;37(5):1202-19.

41. Wang J, Zhang C, Wei X, Blagosklonov O, Lv G, Lu X, et al. TGF- $\beta$ and TGF- $\beta$ /Smad Signaling in the Interactions between Echinococcus multilocularis and Its Hosts. PLoS ONE. 2013;8(2):e55379.

42. Stärkel P, Sempoux C, Leclercq I, Herin M, Deby C, Desager J-P, et al. Oxidative stress, KLF6 and transforming growth factor- $\beta$ up-regulation differentiate non-alcoholic steatohepatitis progressing to fibrosis from uncomplicated steatosis in rats. J Hepatol. 2003;39(4):538-46.

43. Chu AS, Diaz R, Hui JJ, Yanger K, Zong Y, Alpini G, et al. Lineage tracing demonstrates no evidence of cholangiocyte epithelial-to-mesenchymal transition in murine models of hepatic fibrosis. Hepatology. 2011;53(5):1685-95.

44. Greco D, Kotronen A, Westerbacka J, Puig O, Arkkila P, Kiviluoto T, et al. Gene expression in human NAFLD. Am J Physiol Gastrointest Liver Physiol. 2008;294(5):G1281-G7.

45. Sookoian S, Gianotti TF, Rosselli MS, Burgueño AL, Castaño GO, Pirola CJ Liver transcriptional profile of atherosclerosis-related genes in human nonalcoholic fatty liver disease. Atherosclerosis. 2011;218(2):378-85.

46. Benyon RC, Arthur MJ. Extracellular matrix degradation and the role of hepatic stellate cells. Seminars in liver disease. 2001;21(3):373-84 